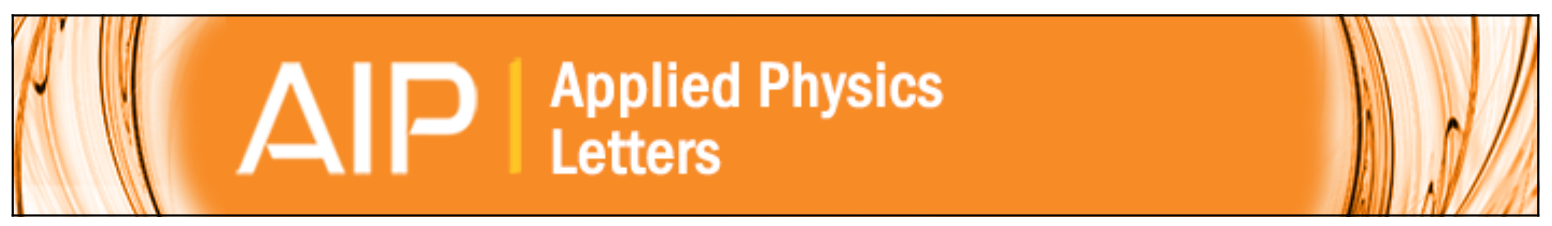

\title{
Spatially optimized gene transfection by laser-induced breakdown of optically trapped nanoparticles
}

Yoshihiko Arita, Maria Leilani Torres-Mapa, Woei Ming Lee, Tomáš ižmár, Paul Campbell, Frank J. Gunn-

Moore, and Kishan Dholakia

Citation: Applied Physics Letters 98, 093702 (2011); doi: 10.1063/1.3554415

View online: http://dx.doi.org/10.1063/1.3554415

View Table of Contents: http://scitation.aip.org/content/aip/journal/apl/98/9?ver=pdfcov

Published by the AIP Publishing

\section{Articles you may be interested in}

Laser-induced fusion of human embryonic stem cells with optical tweezers

Appl. Phys. Lett. 103, 033701 (2013); 10.1063/1.4813822

Pulsed focused ultrasound exposures enhance locally administered gene therapy in a murine solid tumor model

J. Acoust. Soc. Am. 133, 1827 (2013); 10.1121/1.4789390

Role of nuclear tubule on the apoptosis of HeLa cells induced by femtosecond laser

Appl. Phys. Lett. 96, 223701 (2010); 10.1063/1.3447365

Femtosecond laser-induced cell fusion

Appl. Phys. Lett. 92, 093901 (2008); 10.1063/1.2890070

Generation of calcium waves in living cells by pulsed-laser-induced photodisruption

Appl. Phys. Lett. 79, 1208 (2001); 10.1063/1.1397255

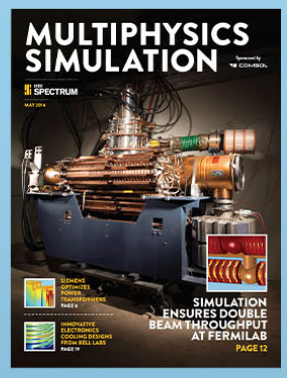




\title{
Spatially optimized gene transfection by laser-induced breakdown of optically trapped nanoparticles
}

\author{
Yoshihiko Arita, ${ }^{1, a)}$ Maria Leilani Torres-Mapa, ${ }^{1}$ Woei Ming Lee, ${ }^{1, b)}$ Tomáš Čižmár, ${ }^{1}$ \\ Paul Campbell, ${ }^{2}$ Frank J. Gunn-Moore, ${ }^{3}$ and Kishan Dholakia ${ }^{1}$ \\ ${ }^{1}$ SUPA, School of Physics and Astronomy, University of St. Andrews, St. Andrews, Fife KY16 9SS, \\ United Kingdom \\ ${ }^{2}$ Carnegie Physics Laboratory and Division of Molecular Medicine, University of Dundee, \\ Dundee DD1 4HN, United Kingdom \\ ${ }^{3}$ School of Biology, Medical and Biological Sciences Building, North Haugh, University of St. Andrews, \\ St. Andrews, Fife KY16 9TF, United Kingdom
}

(Received 20 July 2010; accepted 21 December 2010; published online 2 March 2011)

\begin{abstract}
We demonstrate laser-induced breakdown of an optically trapped nanoparticle with a nanosecond laser pulse. Controllable cavitation within a microscope sample was achieved, generating shear stress to monolayers of live cells. This efficiently permeabilize their plasma membranes. We show that this technique is an excellent tool for plasmid-DNA transfection of cells with both reduced energy requirements and reduced cell lysis compared to previously reported approaches. Simultaneous multisite targeted nanosurgery of cells is also demonstrated using a spatial light modulator for parallelizing the technique. (C) 2011 American Institute of Physics.

[doi:10.1063/1.3554415]
\end{abstract}

The formation of cavitation bubbles in the vicinity of living cells can strongly influence their plasma membrane permeability. ${ }^{1-5}$ It is recognized that acoustic bubbles driven by ultrasonic irradiation (insonation) can lead to enhanced membrane permeabilization (sonoporation) of cells., ${ }^{2,3}$ When cavitation occurs near a rigid boundary such as a cell membrane, the bubbles tend to collapse asymmetrically, often forming high speed liquid jets directed toward the wall. ${ }^{6}$ This can cause localized membrane poration, thus allowing the uptake of external molecules by these cells. However, this approach often leads to non-uniform and sporadic molecular uptake that lacks refined spatial control. In parallel, a number of laser-based methods have been established that facilitate transient poration of the lipid bilayer of a cell for the introduction of therapeutic agents. Such optical methods add the potential of sterility and reconfigurability and typically offer single cell targeting. ${ }^{7}$

Optical methods can also induce cavitation by the optical breakdown or ablation of an absorptive medium ${ }^{8-10}$ using high power nanosecond laser sources. Of the approaches devised to date however, membrane permeabilization of a zone of cells has been achieved at the expense of a significant level of cell lysis owing to the relatively high breakdown threshold of the absorptive substances, e.g. water (present in the buffer medium) or silica (glass coverslips). This results in a much larger cavitation bubble compared to the cell size that effectively reduces cell viability. ${ }^{11-13}$

In this letter, we demonstrate targeted membrane permeabilization of multiple cells within a specific and controllable area utilizing cavitation bubbles excited by the laser-induced breakdown (LIB) of one or more optically trapped nanoparticles. When light from a nanosecond pulsed laser is focused onto an optically trapped nanoparticle, LIB can take place, ${ }^{14}$

\footnotetext{
${ }^{\text {a) }}$ Author to whom correspondence should be addressed. Electronic mail: ya10@st-andrews.ac.uk.

${ }^{b)}$ Present address: Harvard Medical School, Massachusetts General Hospital, Wellman Center for Photomedicine, 50 Blossom St., BAR 814, Boston, MA 02114, USA.
}

leading to the formation of plasma and emission of shockwaves by its expansion followed by the vaporization of the nanoparticle or liquid (surrounding aqueous medium). ${ }^{15}$ This vapor volume effectively constitutes a cavitation bubble, which expands as the volume of the ablated nanoparticle or vaporized liquid increases. Bubble expansion and its subsequent collapse can be accompanied by the emission of acoustic transients and microjetting ${ }^{16}$ depending on the position of the cavitation bubble relative to the substrate. These photomechanical properties can lead to localized membrane permeabilization over multiple cells within a well-defined target zone. $^{5}$

Optical tweezers allow the confinement and positioning of micro- and nano-particles at a desired location within the buffer medium. Therefore, using this tool for the LIB of nanoparticles, we can gain additional degrees of freedomthe nanoparticle material, its size, and the LIB position relative to the cell monolayer. In this study, we show that these additional parameters offer a method to minimize the energy required for controllable cavitation, which leads to the permeabilization or transfection of cells in a targeted area, while reducing cell lysis.

The technique is shown to achieve cell transfection of a plasmid expressing Mito-DsRed with a modest pulse energy of $\leq 1 \mu \mathrm{J}$. This is typically an order of magnitude smaller than for previously reported studies ${ }^{12,13}$ which utilized the breakdown of the surrounding buffer medium in which cells are cultured. Furthermore, we parallelized the process to allow simultaneous multisite targeting nanosurgery of cells using a spatial light modulator (SLM) for multipoint nanoparticle trapping as well as the multiplexing of the nanosecond laser beam for LIB of individual nanoparticles located in a spatially extended array of optical traps.

In our system, a $1070 \mathrm{~nm} \mathrm{CW}$ fiber laser (IPG Laser $\mathrm{GmbH}$, YLM-5-1070-LP: power of $\sim 100 \mathrm{~mW}$ ) was focused by a microscope objective (Nikon Ltd., E plan $100 \times 1.25$ numerical aperture (NA)/oil) to optically trap a polystyrene nanoparticle (from $400 \mathrm{~nm}$ to $2 \mu \mathrm{m}$ in diameter) within the 

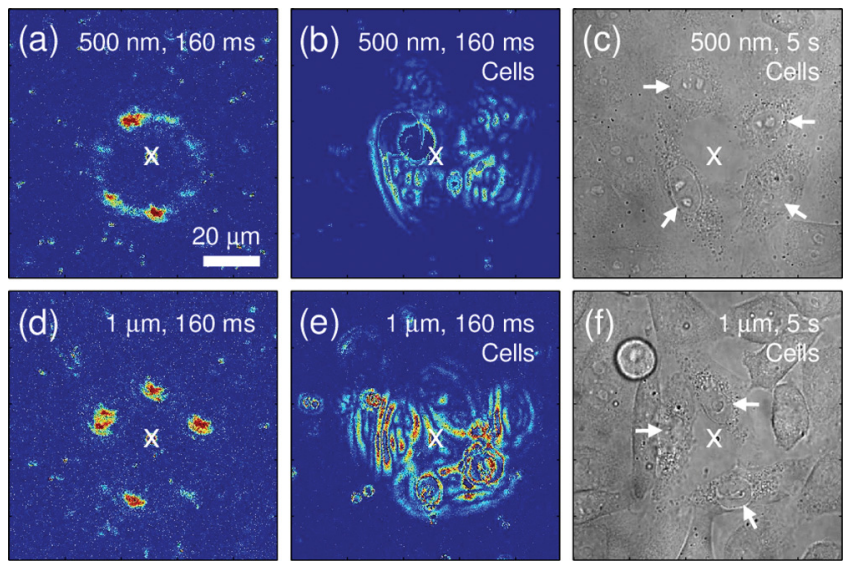

FIG. 1. (Color online) Formation of residual gas bubbles and cellular displacements by nanoparticle LIB. [(a) and (d)] Residual gas bubbles formed in a circle over $\sim 160 \mathrm{~ms}$ after the LIB of $500 \mathrm{~nm}$ [video 1] [URL: http:// dx.doi.org/10.1063/1.3554415.1] and $1 \mu \mathrm{m}$ [video 3] [URL: http:// dx.doi.org/10.1063/1.3554415.3] nanoparticles. [(b) and (e)] Nanoparticle LIB in the presence of CHO-K1 cells indicating cellular displacements over $\sim 160 \mathrm{~ms}$ after the LIB of $500 \mathrm{~nm}$ [video 2] [URL: http://dx.doi.org/ 10.1063/1.3554415.2] and $1 \mu \mathrm{m}$ [video 4] [URL: http://dx.doi.org/10.1063/ 1.3554415.4] nanoparticles. [(c) and (f)] Cell morphology, $5 \mathrm{~s}$ after the LIB of $500 \mathrm{~nm}$ and $1 \mu \mathrm{m}$ nanoparticles (enhanced online).

buffer medium. The nanoparticle was manipulated to a predefined location between 0 and $25 \mu \mathrm{m}$ above the plane of the cell monolayer. A $532 \mathrm{~nm}$ frequency doubled Q-switched Nd:YAG laser (Elforlight Ltd., SPOT: pulse width of $\sim 1 \mathrm{ns,}$ energy of $\leq 1 \mu \mathrm{J}$, and repetition rate of $1 \mathrm{kHz}$ ) was coaligned with the trapping beam and focused through the same objective onto the trapped nanoparticle for $40 \mathrm{~ms}$ to effect LIB (Fig. 1 of the supplementary material ${ }^{20}$ ).

Initial experiments explored the formation of residual or non-condensable gas bubbles and cellular responses as a direct consequence of the LIB of nanoparticles. ${ }^{17}$ Images of these events were recorded by an electron-multiplying charge-coupled device (EMCCD) camera [Andor Technology plc., iXonEM+885, 31.23 frames per second (fps)] coupled to the microscope. The sum of the absolute values obtained from the gradient signals at each pixel between six consecutive frames over $\sim 160 \mathrm{~ms}$ before and after the LIB (supplementary MATLAB ${ }^{\circledR} \operatorname{code}^{20}$ ) revealed the formation of residual gas bubbles [Figs. 1(a) and 1(d)] and cellular displacements [Figs. 1(b) and 1(e)]. Here, LIB of single nanoparticles of $500 \mathrm{~nm}$ in diameter [Fig. 1(a)] and $1 \mu \mathrm{m}$ in diameter [Fig. 1(d)], trapped at displacements of $10 \mu \mathrm{m}$ from the surface of the coverslip substrate, illustrates the radial pattern of gas bubbles which is centered on the site of the initial LIB [indicated as " $x$ " in Figs. 1(a) and 1(d)]. These gas bubbles are a likely consequence of secondary bubbles formed by the interaction between the laser-induced cavitation bubble and substrate ${ }^{18}$ (Fig. 2 of the supplementary material $\left.^{20}\right)$. The circles of gas bubbles typically extended to diameters of $\sim 35 \mu \mathrm{m}$ [Fig. 1(a)] and $\sim 40 \mu \mathrm{m}$ [Fig. 1(d)] for the $500 \mathrm{~nm}$ and $1 \mu \mathrm{m}$ nanoparticles, respectively.

In the presence of a CHO-K1 cell monolayer upon the coverslip, the circular area affected by the LIB of a $500 \mathrm{~nm}$ nanoparticle was typically smaller, $\sim 40 \mu \mathrm{m}$ in diameter [Fig. 1(b)], than that by the $1 \mu \mathrm{m}$ particle LIB, $\sim 60 \mu \mathrm{m}$ in diameter [Fig. 1(e)]. A large displacement of cells from the LIB of a $1 \mu \mathrm{m}$ particle, was indicated by an intense signal obtained through implementation of a tracking algorithm

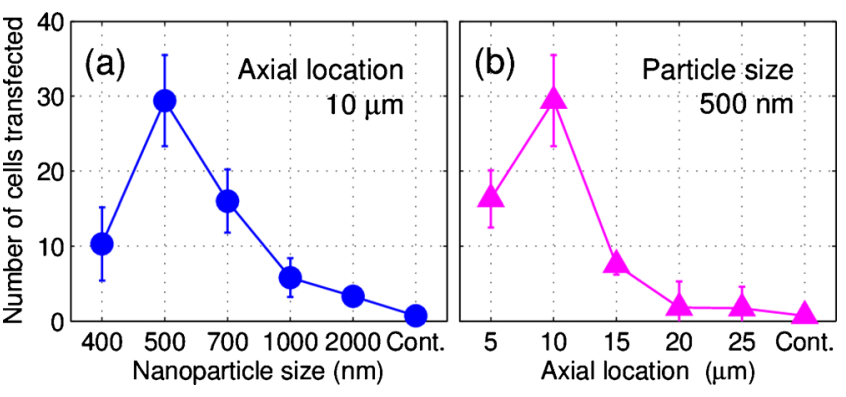

FIG. 2. (Color online) Optimization of transfection of Mito-DsRed encoding plasmids into multiples of $\mathrm{CHO}-\mathrm{K} 1$ cells by LIB parameters. (a) Number of cells transfected with different nanoparticle sizes at the fixed axial location of $10 \mu \mathrm{m}$. (b) Number of cells transfected at different axial locations with the fixed particle size of $500 \mathrm{~nm}$. The error bars indicate $2 \sigma$.

[Fig. 1(e) and supplementary MATLAB ${ }^{\circledR} \operatorname{code}^{20}$ ] resulting in three or more cells undergoing lysis [arrows in Fig. 1(f)], while cell morphology with the $500 \mathrm{~nm}$ nanoparticle LIB was characterized by granulation and nucleus condensation [arrows in Fig. 1(c)].

In these experiments, the temporal resolution is insufficient to observe initial LIB events including cavitation bubble formation and collapse. However, it is assumed that a single cavitation bubble, centered on the site of the nanoparticle LIB, is initiated in the same manner as LIB of a liquid. Any initial cavitation bubble generated by LIB of the nanoparticle prior to the circles of residual gas bubbles is not observed and is assumed to be ultrafast in growth and decay. Thus, the mechanism suggests liquid LIB using a Q-switched nanosecond laser ${ }^{19}$ can be extended to the present data. The cavitation bubble can interact with the substrate via asymmetric collapse accompanied by hydrodynamic jets toward the surface depending on the dimensionless stand-off parameter $\gamma$, which is defined by the position of the cavitation bubble relative to the substrate: ${ }^{18}$

$$
\gamma=\mathrm{Z}_{0} / \mathrm{R}_{\max },
$$

where $\mathrm{Z}_{0}$ is the normal distance from the substrate to the center of the cavitation bubble and $R_{\max }$ is the maximum radius of the bubble [Figs. 2(a) and 2(b) of the supplementary material $\left.{ }^{20}\right]$. The kinetic jets have the potential to damage the surface, which leads to the appearance of circular clearance zones in the cell covered substrates [Figs. 1(c) and 1(f)]. The subsequent radial flow along the surface can be accompanied by secondary bubbles at the leading edge of the radial flow [Fig. 2(c) of the supplementary material ${ }^{20}$ ]. The secondary bubbles can lead to the residual gas bubbles, which persist more than milliseconds.

In previous studies on LIB of liquid, ${ }^{18,19}$ the maximum bubble radius $R_{\max }$ was found to scale with the overall diameter of the damaged area. The initial observations of nanoparticle LIB found principally that the circles of residual gas bubbles are formed on the cell-free substrates [Figs. 1(a) and 1(d)], that circular clearance zones also appear to form when cells are present [Figs. 1(c) and 1(f)], and that the effects are rather localized $\leq 20 \mu \mathrm{m}$ in radius (i.e. the maximum bubble radius $R_{\max }$ ). It is reasonable to infer that any initial cavitation bubble formed from the LIB of a nanoparticle has a probable stand-off parameter that lies within the range, 0.5 $\leq \gamma \leq 0.6$ by substituting for $\mathrm{Z}_{0}=10 \mu \mathrm{m}$ and for $\mathrm{R}_{\max }$ $\sim 20 \mu \mathrm{m}$ (for a $1 \mu \mathrm{m}$ particle) and $\sim 17.5 \mu \mathrm{m}$ (for a 500 nm particle) into Eq. (1). Note that a cavitation bubble form- 


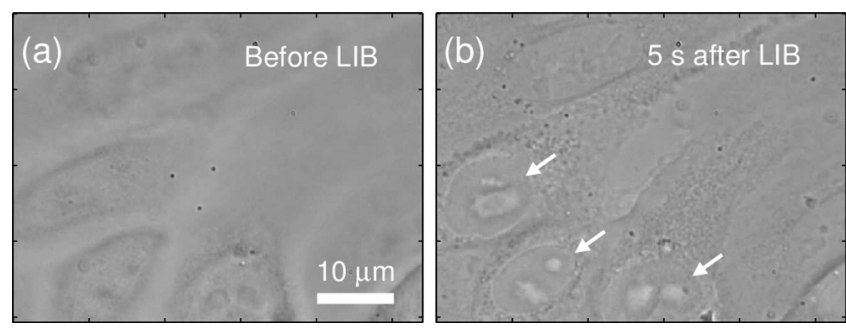

FIG. 3. Simultaneous multisite targeted nanosurgery of cells using SLMs [video 5] [URL: http://dx.doi.org/10.1063/1.3554415.5]. (a) Three trapped $500 \mathrm{~nm}$ nanoparticles placed on an equilateral triangle with side of $\sim 10 \mu \mathrm{m}$ positioned at $\sim 10 \mu \mathrm{m}$ above the cell monolayer before LIB. (b) Cell morphology, $5 \mathrm{~s}$ after the simultaneous LIB of the three trapped nanoparticles (enhanced online)

ing with $\gamma<1$ has the maximum radius $\mathrm{R}_{\max }$ greater than $\mathrm{Z}_{0}$, so that the bubble boundary is already in "contact" with the substrate.

Here, we observed that the physical impact to cells in a targeted area was dependent on the nanoparticle size. Thus, the shear stress induced membrane permeabilization of cells in a defined zone can be optimized by the LIB parameters, such as the nanoparticle size and its location relative to the cell monolayer at which LIB is performed, i.e. by optimizing the stand-off parameter $\gamma$.

Transfection of Mito-DsRed encoding plasmids into multiples of HEK 293 cells was first achieved by the LIB of $500 \mathrm{~nm}$ nanoparticles at an axial location of $10 \mu \mathrm{m}$ (supplementary methods ${ }^{20}$ and Fig. 3 of the supplementary materia $\left.{ }^{20}\right)$. The optimization of this technique for gene transfection was next studied using two different parameters, namely, nanoparticle size, ranging from $400 \mathrm{~nm}$ to $2 \mu \mathrm{m}$ in diameter [Fig. 2(a)], and axial location, between 5 and $25 \mu \mathrm{m}$ from the plane of the cell monolayer [Fig. 2(b)]. Here, the dependence on nanoparticle size may determine the volume of ablated material, which in turn dictates the size of the cavitation bubble. LIB from a range of heights above the cell monolayer also provided a means of optimizing molecular delivery since the shear stress to cells from the nanoparticle LIB is dependent on the stand-off parameter $\gamma$ [Eq. (1) and Fig. 2 of the supplementary material $\left.{ }^{20}\right]$. The optimum parameters for cellular transfection were found to be $500 \mathrm{~nm}$ in nanoparticle size and $10 \mu \mathrm{m}$ in axial location for our experimental setup (Fig. 2). The number of transfected cells was typically $\sim 30$ for ten breakdowns per sample at this optimum condition. The control cells were exposed to the $\mathrm{CW}$ and nanosecond lasers, with nanoparticles of $500 \mathrm{~nm}$ in the buffer medium, but without any LIB. Therefore, this technique clearly shows gene transfection of multiple cells in a spatially selective zone while reducing cell lysis by the optimization of two parameters, nanoparticle size and its height above the cell monolayer at which LIB is performed.

Finally we explored the potential to perform simultaneous multisite targeted nanosurgery of cells by the simultaneous LIB of multiple trapped nanoparticles using two SLMs (Hamamatsu, LCOS-SLM X10468). The 1064 nm CW laser (IPG Photonics Ltd.) (for trapping) and the $532 \mathrm{~nm}$ nanosecond laser (for LIB) were both multiplexed by the SLMs to three spots each (Fig. 4 of the supplementary material ${ }^{20}$ ) forming an equilateral triangle with side of $\sim 10 \mu \mathrm{m}$, projected onto the same focal plane of a water immersion objective (Carl Zeiss Ltd., $63 \times 1.2 \mathrm{NA} / \mathrm{W})$. The three trapped nanoparticles, each $500 \mathrm{~nm}$ in diameter, were then targeted simultaneously for breakdown by the multiplexed nanosecond laser [Fig. 3(a)]. Images of multisite LIB were recorded by a charge-coupled device (CCD) camera (Basler piA640$210 \mathrm{gm}, 633 \mathrm{fps})$. The cell morphology, $5 \mathrm{~s}$ after the simultaneous LIB of the three trapped nanoparticles, is characterized by granulation and nucleus condensation [arrows in Fig. 3(b)]. This study suggests that the consolidation of the optical trapping and nanoparticle LIB, with the aid of the SLM technique, has the potential to allow fully optically controlled membrane permeabilization of cells within welldefined multitarget zones.

Here we have described an optical technique that uses LIB of one or more optically trapped nanoparticles to facilitate the optimization of the energy required for controllable cavitation. This was achieved by varying the nanoparticle size and its height above the cell monolayer at which LIB was performed and led to the permeabilization or transfection of cells in a targeted area with retention of viability. This method was readily combined with the use of a SLM and provided a powerful platform for simultaneous multisite nanosurgery of cells.

In conclusion, our LIB approach has the potential to increase the applicability of optically controlled cell transfection. The technique only requires a modest nanosecond laser pulse energy, reduces cell lysis, and operates in a spatially selective manner. It offers major advantages due to its simplicity, lower cost, and higher reliability when compared to existing methods.

We thank the UK EPSRC for funding. K.D. is a Royal Society Wolfson-Merit Award Holder. P.C. acknowledges the MRC for a Discipline Hopping Fellowship and Milstein Award. M.L.T.-M. acknowledges the support of a SUPA Prize Studentship.

${ }^{1}$ J. P. Dear, J. E. Field, and A. J. Walton, Nature (London) 332, 505 (1988). ${ }^{2}$ M. Ward, J. R. Wu, and J. F. Chiu, J. Acoust. Soc. Am. 105, 2951 (1999). ${ }^{3}$ D. L. Miller and J. M. Song, Ultrasound Med. Biol. 29, 887 (2003).

${ }^{4}$ P. Prentice, A. Cuschieri, K. Dholakia, M. Prausnitz, and P. Campbell, Nat. Phys. 1, 107 (2005)

${ }^{5}$ C. D. Ohl, M. Arora, R. Ikink, N. de Jong, M. Versluis, M. Delius, and D. Lohse, Biophys. J. 91, 4285 (2006).

${ }^{6}$ T. B. Benjamin and A. T. Ellis, Philos. Trans. R. Soc. London, Ser. A 260, 221 (1966).

${ }^{7}$ D. J. Stevenson, F. J. Gunn-Moore, P. Campbell, and K. Dholakia, J. R. Soc., Interface 7, 863 (2010).

${ }^{8}$ E. F. Carome, C. E. Moeller, and N. A. Clark, J. Acoust. Soc. Am. 40, 1462 (1966)

${ }^{9}$ C. E. Bell and J. A. Landt, Appl. Phys. Lett. 10, 46 (1967).

${ }^{10}$ P. A. Barnes and K. E. Rieckhof, Appl. Phys. Lett. 13, 282 (1968).

${ }^{11}$ J. S. Soughayer, T. Krasieva, S. C. Jacobson, J. M. Ramsey, B. J. Tromberg, and N. L. Allbritton, Anal. Chem. 72, 1342 (2000).

${ }^{12}$ K. R. Rau, P. A. Quinto-Su, A. N. Hellman, and V. Venugopalan, Biophys. J. 91, 317 (2006)

${ }^{13}$ A. N. Hellman, K. R. Rau, H. H. Yoon, and V. Venugopalan, J. Biophotonics 1, 24 (2008)

${ }^{14}$ H. X. Chen and G. Diebold, Science 270, 963 (1995).

${ }^{15}$ M. R. Prausnitz, P. Chakravarty, W. Qian, and M. A. El-Sayed, J. Acoust. Soc. Am. 127, 1940 (2010).

${ }^{16}$ Y. Tomita and A. Shima, Shock Focusing Effect in Medical Science and Sonoluminescence, 1st ed. (Springer, Berlin, 2003), Chap. 4.

${ }^{17}$ P. K. Kennedy, D. X. Hammer, and B. A. Rockwell, Prog. Quantum Electron. 21, 155 (1997).

${ }^{18}$ Y. Tomita and A. Shima, J. Fluid Mech. 169, 535 (1986).

${ }^{19}$ A. Philipp and W. Lauterborn, J. Fluid Mech. 361, 75 (1998).

${ }^{20}$ See supplementary material at http://dx.doi.org/10.1063/1.3554415 for supplementary methods, figures, videos, and MATLAB ${ }^{\circledR}$ code. 